Research Paper

\title{
Increased Co-Expression of Macrophage Migration In- hibitory Factor and Matrix Metalloproteinase 9 Is As- sociated with Tumor Recurrence of Meningioma
}

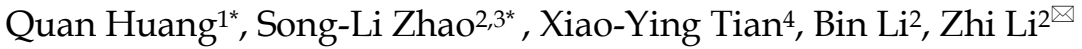 \\ 1. Department of Neurosurgery, The First Affiliated Hospital, Sun Yat-sen University. 58 Zhongshan Road II, Guangzhou 510080, China; \\ 2. Department of Pathology, The First Affiliated Hospital, Sun Yat-sen University. 58 Zhongshan Road II, Guangzhou 510080, China; \\ 3. Department of Pathology, Nanhai Affiliated Hospital of Southern Medical University. 40 Foping Road, Foshan 528200, China; \\ 4. School of Chinese Medicine, Hong Kong Baptist University, Hong Kong, China. \\ ${ }^{*}$ Co-first authors.
}

$\triangle$ Corresponding author: Dr. Zhi Li, Department of Pathology, The First Affiliated Hospital, Sun Yat-sen University. 58 Zhongshan Road II, Guangzhou 51008, CHINA. Tel: (8620) 87330890 Fax: (8620) 87331780 E-mail: lizhi@mail.sysu.edu.cn.

( ) Ivyspring International Publisher. This is an open-access article distributed under the terms of the Creative Commons License (http://creativecommons.org/ licenses/by-nc-nd/3.0/). Reproduction is permitted for personal, noncommercial use, provided that the article is in whole, unmodified, and properly cited.

Received: 2012.09.07; Accepted: 2012.12.27; Published: 2013.01.24

\begin{abstract}
Background and Objective: We detected the expression of MIF and matrix metalloproteinase 9 (MMP9) in meningiomas to determine whether they are valuable recurrence predictor for meningioma.

Methods: 67 cases of meningiomas, including 57 benign tumors (WHO grade I) and 10 non-benign tumors (WHO grade II and III), were collected, and expression of MIF and MMP9 in tissue microarray was evaluated immunohistochemically. The correlations between immunostainings and clinicopathological parameters, as well as the follow-up data of patients, were analyzed statistically.

Results: Increased expressions of both MIF (58.2\%, 39/67) and MMP9 $(55.2 \%, 37 / 67)$ were significantly associated with microvessel density (MVD) of tumor, but only dual high-expression of MIF and MMP9 was in relation to tumor invasion $(P=0.016)$ and tumor recurrence $(P=0.00 I)$. Based on univariate analysis, histological grade, tumor invasion and co-expression of MIF and MMP9 were significant predictors for recurrence. However, only histological grade and co-expression of MIF and MMP9 in tumor were independent recurrence factors with a hazard ratio of $49.033(\mathrm{P}=0.002)$ and $37.766(\mathrm{P}=0.002)$ in multivariate analysis.

Conclusions: Together with histological grade, increased co-expression of MIF and MMP9 in tumor might be a valuable predictor for recurrence, especially for benign meningiomas.
\end{abstract}

Key words: Meningioma; Macrophage migration inhibitory factor (MIF); Matrix metalloproteinase 9 (MMP9); Recurrence.

\section{Introduction}

Meningiomas occur most commonly in middle-aged patients and account for about $24-30 \%$ of primary intracranial tumors. Histological grade is the most useful morphologic predictor of recurrence of meningiomas [1]. While meningiomas with grade I have recurrence rates of about $7-25 \%$, atypical men- ingiomas (grade II) recur in $29-52 \%$ of cases and anaplastic meningiomas (grade III) at rates of 50-94\% [2]. However, except for the histological grading of tumors, other risk factors for recurrence are not well understood in the cases with benign meningioma. In clinical, more than $90 \%$ of meningiomas are benign 
(grade I) [3], and the ideal treatment for these tumors is complete surgical resection but, when invasion of the adjacent structures, including dura mater, bone, and brain parenchyma, is present (these are not prognostic factors in the WHO criteria), this aim is often not achieved. Therefore, the histological grade alone sometimes is inadequate to precisely predict the patients' prognosis, especially in those with benign meningiomas. It is necessary to explore and investigate the more precise recurrence prediction for meningiomas.

Macrophage migration inhibitory factor (MIF) is a multi-functional cytokine associated with inflammation and tumorigenesis [4]. A deregulated cytokine expression pattern is observed during neurological diseases, including brain tumor [5]. Current studies have shown that MIF plays an important role in tumor growth and progression. Increased expression of MIF has been identified in various human cancers, including breast cancer, adenocarcinoma of lung, hepatocellular carcinoma and cervical carcinoma [6-8]. Recent studies also demonstrated that MIF modulates the biological response in different cell types by inducing the nuclear factor-kappa B (NF- $\kappa \mathrm{B})$, Erk1/2 and activating protein-1(AP-1) signaling pathways, or by binding CD74/CD44 receptor complex [9-11]. In brain tumor, MIF is one of the most up-regulated transcripts in glioblastomas [12-13]. More recently, MIF has been shown to promote cell proliferation and migration/invasion in prostate cancer, lung adenocarcinoma, and neuroblastoma cells [14-15]. However, the relationship between MIF and meningiomas has not been clarified yet.

Matrix metalloproteinase 9 (MMP9) can degrade extracellular matrix (ECM) and basement membrane (BM), thus playing an essential role in invasion and metastasis [16]. Previous studies have associated MMP9 with the invasion and metastasis of a large variety of cancers, such as lung, prostate, breast, and colon cancers [17-18]. In meningiomas, MMP9 expression in tumor has been correlated with tumor edema and cell proliferation, both of which are predictive factors for tumor recurrence [19]. Recent studies have also reported that MIF can induce MMP9 expression by MEK-ERK MAP kinase pathway [20], and tumor-derived MIF promoting release of MMP9 in neutrophils contributes to tumor progression of head and neck cancer [21]. Our previous study also revealed MIF-induced MMP9 enhanced cell invasion in nasopharyngeal carcinoma cells [22]. These findings suggest that MIF and MMP9 may play a co-operative role in biological behavior of tumors. However, no study has been conducted to investigate this in meningiomas.
The objective of this study was to analyze semiquantitatively the expression of MIF and MMP9, as well as intratumoral microvessel density in the tumor. We wonder if their expression status was in correlation with tumor grade and recurrence of meningiomas. We hypothesized that MIF might play roles in the recurrence of meningiomas by modulating tumor cell invasion and angiogenic activity of tumors. Our main goal was to estimate the risk of meningioma recurrence via histopathological findings and biomarkers during early postoperative period.

\section{Material and Methods}

\section{Patients and clinicopathological data}

Sixty-seven primary intracranial meningioma patients were admitted to the First Affiliated Hospital of Sun Yat-sen University during the period of 1999 to 2009. The patients were followed from 3 to 110 months, with a mean period of 56 months, and 14 patients $(20.1 \%)$ had recurrence of tumor during the follow-up period. None patients had received prior radiotherapy before surgery. The extent of tumor removal was graded according to Simpson's criteria [23], and all of patients recruited in this study underwent tumor excision of Simpson Grade I (complete tumor excision including the dural attachment). The size of the tumors was categorized as large $(>4 \mathrm{~cm})$ or small $(<4 \mathrm{~cm})$ by calculating its mean diameter on MRI [24]. After surgery, the tumor samples were fixed with $10 \%$ neutral-buffered formalin and paraffin-embedded. Histological classification and grading of each resected specimens were reviewed by an authoritative pathologist according to WHO criteria.

\section{Paraffin tissue microarray construction}

In this study, the paraffin tissue microarray construction was conducted as routine method. Briefly, a section was cut from each paraffin block and stained with hematoxylin and eosin (H\&E). Each donor block was overlaid with the corresponding H\&E slide and observed by experienced pathologists. The area in the donor block for tissue microarray sampling was verified according to the H\&E slide and marked. A manual tissue arrayer (Beecher Instruments Inc., USA) was used for array construction. Three representative 1.0-mm cores were removed from each donor block and transferred to a premolded recipient paraffin block with designated orientation. An additional six cores derived from tonsil, lymph node, and breast carcinoma was used as control material. Serial sections with 4 um thickness were cut from the arrayed block and mounted on aminopropyltriethoxysilane (APES)-coated glass slides and stored at $4^{\circ} \mathrm{C}$ for fur- 
ther analysis.

\section{Immunohistochemical staining and scoring analysis}

The tissue array sections were subjected to immunostaining using a ChemMate Envision/HRP kit (Dako Co., Denmark). Slides were dewaxed and rehydrated routinely before antigen retrieval by $\mathrm{mi}-$ crowave. Non-specific binding sites were blocked with a solution of $2 \%$ bovine serum albumin. The slides were incubated with diluted primary antibodies (MIF mouse monoclonal antibody 1:100, Abcam, USA; MMP9 mouse monoclonal antibody 1:50 and CD34 mouse monoclonal antibody 1:100, Santa Cruz Biotechnology, USA) at $4^{\circ} \mathrm{C}$ overnight. EnVision system was used to detect these antigens in meningiomas. The slides were developed with DAB, and lightly counterstained with hematoxylin.

The immunohistochemical reactions were analyzed by two individuals and followed the criteria [19]: 0) negative, 1) $10 \%$ up to $20 \%$, 2) $21 \%$ up to $50 \%$, and 3) $51 \%$ or more. Immunoreactive intensity was graded as 0) negative, 1) weakly yellowish, 2) brown-yellow, 3) dark brown. The multiplier of the above two scores was defined as the protein expression in tumor and the scale was given a numerical value from 0 to 9 for further analysis. MIF or MMP9 high-expression in the tumor was defined when the score was equal or more than 4, whereas low-expression of proteins was less than score 4.

\section{Microvessel density (MVD) counting and im- age analysis}

Intratumoral microvessel density (MVD) was highlighted in the intact tumor sections by staining endothelial cells for anti-CD34. The most intense neovascularization or hotspots in tumor was firstly determined by light microscopy. Images were captured and analyzed using a CCD camera and Image ProPlus software. The MVD was calculated by selecting the area with the highest vascular density and five medium power fields $(200 \times)$ were captured and the microvessels counted. Vessels between 8 and $14 \mathrm{um}$ were regarded as microvessels, in accordance with the criteria in a previous study [25].

\section{Statistical analysis}

All statistical analyses were carried out using SPSS 13.0 software for Windows. The chi-square test was used to assess MIF and MMP9 expression with clinicopathological characteristics. Univariate analysis by Student's $t$ test was used to assess protein expression in relation to angiogenesis of meningioma. The recurrence curve of patients was determined using the
Kaplan-Meier method and Cox regression, and statistical evaluation was performed using the log rank test. A P-value $<0.05$ was considered statistically significant.

\section{Results}

\section{Clinicopathological findings of patients with meningiomas}

The patients were 17 males and 50 females with a median age of 45 years old. 57 patients had grade I meningiomas, including 26 meningothelial, 15 fibrous, 13 transitional and 3 psammomatous variant. 6 patients had grade II meningiomas, including 5 atypical and 1 clear cell variant. The other 4 patients had grade III meningiomas, including 2 anaplastic, 1 papillary and 1 rhabdoid variant. All of cases were classified as Simpson grade I in which complete tumor excision including the dural attachment was achieved. Based on the MRI examination, 43 cases were categorized as small tumor and 24 were large tumor. 9 cases of non-benign meningiomas (grade II and III, 90\%, 9/10) were found to invade the dura matter and cortical parenchyma, but only $11(19.3 \%, 11 / 57)$ benign meningiomas (grade I) were found to invade the adjacent dura matter, skull and (or) brain parenchyma. Of the 67 patients, 14 patients developed a recurrent meningioma during the period of following-up, including 7 benign tumors and 7 non-benign tumors. The mean time to recurrence was $58.3 \pm 7.8$ months (range, 48 to $64 \mathrm{mos}$ ) for benign meningiomas and $35.6 \pm 22.5$ months (range 3 to $65 \mathrm{mos}$ ) for non-benign meningiomas.

\section{MIF and MMP9 expression in correlation to clinicopathological parameters and MVD in meningiomas}

A total of $58.2 \%$ of tumor tissues (39/67) showed high expression for MIF. MIF expression was intense in the cytoplasm of tumor cells (Figure 1A, B). MIF high expression was significantly correlated with MVD of tumor and high expression of MMP9 in tumor, but not correlated with tumor invasion and recurrence. MMP9 immunoreactivity was also detected in the tumor cells (Figure 1C). High expression of MMP9 $(55.2 \%, 37 / 67)$ was found to have a positive significant correlation with MVD of tumor (Figure 1D). In our study, we found that MIF and MMP9 could be detected in a concomitant high-expression in 28 tumors. MIF and MMP9 showed a co-expression in the same portion of tumor cells on serial sections (Figure 1E). The dual high-expression of MIF and MMP9 exhibited closely correlation with tumor invasion and recurrence (P values were less than 0.05, Ta- 
ble 1). We examined the relationship between other clinicopathological variables and MIF/MMP9 ex- pression or MVD in tumor; no significant correlations were found.
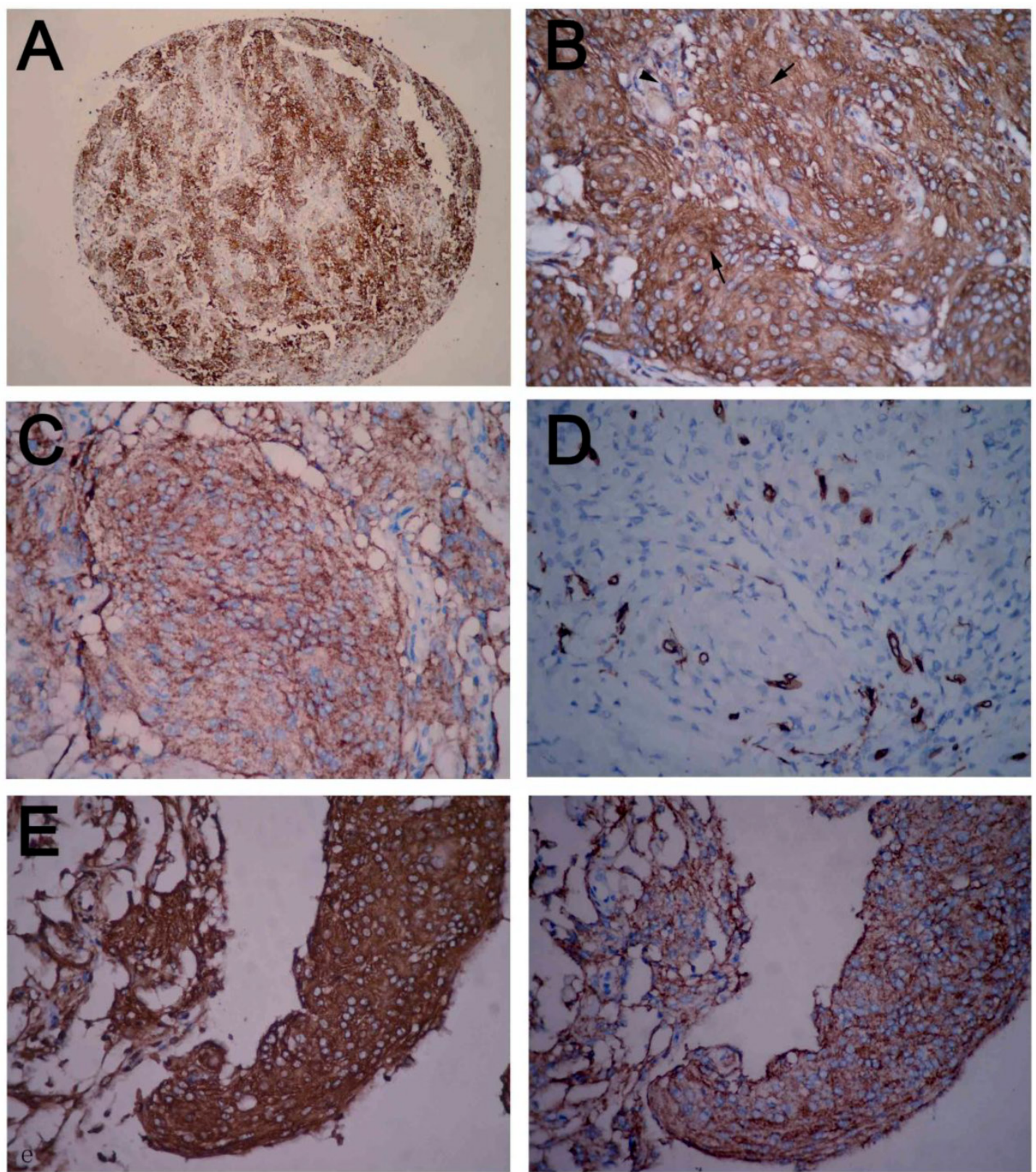

Figure I. Immunohistochemical staining of meningioma tissue microarray. (A) Positive MIF staining in low-power field. (B) Strongly positive cytoplasmic MIF staining in tumor cells and weak staining of stromal cells of meningioma. (C) Diffuse positive MMP9 staining in tumor cells. (D) Microvessels in tumor were highlighted by staining endothelial cells. (E) MIF and MMP9-positive tumor cells in the same area of tumor in serial section. MIF-positive cells on the left, MMP9 positive cells on the right (A, immunohistochemical staining with original magnification, $100 \times$; B-E, immunohistochemical staining with original magnification, 400x). 
Table I. Correlation among proteins expression, MVD value and clinicopathological parameters of patients with meningioma.

\begin{tabular}{|c|c|c|c|c|c|c|c|}
\hline \multirow[t]{2}{*}{ Variable } & \multicolumn{2}{|c|}{ MIF expression a) } & \multicolumn{2}{|c|}{ MMP9 expression a) } & \multicolumn{2}{|c|}{ Co-expression of MIF and MMP9 a) } & \multirow{2}{*}{$\begin{array}{l}\text { MVD Value b) } \\
(\text { mean } \pm \text { SD) }\end{array}$} \\
\hline & $\begin{array}{l}\text { Low exp. } \\
(\mathrm{n}=28)\end{array}$ & $\begin{array}{l}\text { High exp. } \\
(\mathrm{n}=39)\end{array}$ & $\begin{array}{l}\text { Low exp. } \\
(n=30)\end{array}$ & $\begin{array}{l}\text { High exp. } \\
(\mathrm{n}=37)\end{array}$ & $\begin{array}{l}\text { Non-dual high } \\
\text { exp. }(n=39)\end{array}$ & $\begin{array}{l}\text { Dual high exp. } \\
(\mathrm{n}=28)\end{array}$ & \\
\hline \multicolumn{8}{|l|}{ Age (year) } \\
\hline$<45(n=33)$ & 15 & 18 & 14 & 19 & 20 & 13 & $17.51 \pm 6.22$ \\
\hline \multirow[t]{2}{*}{$\geq 45(\mathrm{n}=34)$} & 13 & 21 & 16 & 18 & 19 & 15 & $17.95 \pm 7.21$ \\
\hline & $\mathrm{P}=0.424$ & & $\mathrm{P}=0.686$ & & $\mathrm{P}=0.612$ & & $\mathrm{P}=0.789$ \\
\hline \multicolumn{8}{|l|}{ Gender } \\
\hline Male $(\mathrm{n}=17)$ & 6 & 11 & 9 & 8 & 11 & 6 & $16.41 \pm 5.74$ \\
\hline \multirow[t]{2}{*}{ Female $(n=50)$} & 22 & 28 & 21 & 29 & 28 & 22 & $18.19 \pm 6.97$ \\
\hline & $\mathrm{P}=0.317$ & & $\mathrm{P}=0.267$ & & $\mathrm{P}=0.317$ & & $\mathrm{P}=0.347$ \\
\hline \multicolumn{8}{|l|}{ Histological grade } \\
\hline Grade I (n=57) & 25 & 32 & 26 & 31 & 34 & 23 & $17.31 \pm 6.37$ \\
\hline \multirow[t]{2}{*}{ Grade II and III $(n=10)$} & 3 & 7 & 4 & 6 & 5 & 5 & $20.20 \pm 8.19$ \\
\hline & $\mathrm{P}=0.592$ & & $\mathrm{P}=0.819$ & & $\mathrm{P}=0.701$ & & $\mathrm{P}=0.209$ \\
\hline \multicolumn{8}{|l|}{ Tumor size } \\
\hline Small $(n=43)$ & 16 & 27 & 21 & 22 & 25 & 18 & $18.63 \pm 7.04$ \\
\hline \multirow[t]{2}{*}{ Large $(n=24)$} & 12 & 12 & 9 & 15 & 14 & 10 & $16.14 \pm 6.81$ \\
\hline & $\mathrm{P}=0.285$ & & $\mathrm{P}=0.333$ & & $\mathrm{P}=0.998$ & & $\mathrm{P}=0.146$ \\
\hline \multicolumn{8}{|l|}{$\begin{array}{l}\text { Invasion of adjacent } \\
\text { tissue }\end{array}$} \\
\hline No $(n=47)$ & 23 & 24 & 24 & 23 & 32 & 15 & $17.84 \pm 6.89$ \\
\hline \multirow[t]{2}{*}{ Yes $(n=20)$} & 5 & 15 & 6 & 14 & 7 & 13 & $17.50 \pm 6.34$ \\
\hline & $\mathrm{P}=0.096$ & & $\mathrm{P}=0.131$ & & $\mathrm{P}=0.016$ & & $\mathrm{P}=0.851$ \\
\hline \multicolumn{8}{|l|}{ Tumor recurrence } \\
\hline No $(n=53)$ & 25 & 28 & 26 & 27 & 35 & 18 & $18.16 \pm 7.07$ \\
\hline \multirow[t]{2}{*}{ Yes $(n=14)$} & 3 & 11 & 3 & 11 & 4 & 10 & $16.11 \pm 4.88$ \\
\hline & $\mathrm{P}=0.180$ & & $\mathrm{P}=0.268$ & & $\mathrm{P}=0.001$ & & $\mathrm{P}=0.308$ \\
\hline \multicolumn{8}{|l|}{ MIF expression } \\
\hline Low expression $(\mathrm{n}=28)$ & & & 19 & 9 & & & $15.35 \pm 4.36$ \\
\hline \multirow[t]{2}{*}{ High expression $(n=39)$} & & & 11 & 28 & & & $19.45 \pm 7.55$ \\
\hline & & & $\mathrm{P}=0.001$ & & & & $\mathrm{P}=0.012$ \\
\hline \multicolumn{8}{|l|}{ MMP9 expression } \\
\hline Low expression $(n=30)$ & 19 & 11 & & & & & $15.61 \pm 4.14$ \\
\hline \multirow[t]{2}{*}{ High expression $(\mathrm{n}=37)$} & 9 & 28 & & & & & $19.45 \pm 7.83$ \\
\hline & $\mathrm{P}=0.001$ & & & & & & $\mathrm{P}=0.018$ \\
\hline \multirow[t]{2}{*}{ MVD value } & $15.35 \pm 4.36$ & $19.45 \pm 7.55$ & $15.61 \pm 4.14$ & $19.45 \pm 7.83$ & $15.37 \pm 4.01$ & $20.48 \pm 8.21$ & \\
\hline & $\mathrm{P}=0.012$ & & $\mathrm{P}=0.018$ & & $\mathrm{P}=0.001$ & & \\
\hline
\end{tabular}

a). Chi-square test. b).independent-sample $\mathrm{T}$ test

\section{Association of MIF and MMP9 expression with recurrence of meningiomas}

The mean recurrence-free time of patients with benign and non-benign meningiomas was $69.7 \pm 18.3$ months and $41.2 \pm 20.9$ months, respectively. There was a significant difference statistically $(\mathrm{P}=0.001$, Figure 2A). When the tumor invaded the adjacent tissue, the recurrence-free time of patients was significantly shorter $(\mathrm{P}=0.001$, Figure $2 \mathrm{~B})$. However, there was no significant correlation between recurrence-free time of patients and age, gender, tumor size or MVD 
of tumors. In univariate analysis, the recurrence-free time of patients was not significantly different between meningiomas with or without single MIF/MMP9 high-expression $(P>0.05$, Figure $2 \mathrm{C}$ and $2 \mathrm{D})$, but in the patients with dual high-expression of MIF and MMP9 in tumors, the recurrence-free time was significantly shorter than that of patients with non-dual high-expression of proteins (Figure 2E).

In addition, in benign meningiomas, $6(85.7 \%)$ of 7 recurred tumors was found dual high-expression of MIF and MMP9, however, only 1 recurred tumor $(14.3 \%, 1 / 7)$ was found in patients without dual high-expression of proteins. There was a significant correlation between proteins high expression and recurrence-free time of patients (Figure 2F, Table 2). That indicated dual high-expression of MIF and MMP9 was a valuable predictor for the tumor recurrence in patients with histological benign meningiomas. In multivariate analysis, only histological grade and dual high-expression of MIF and MMP9 in meningioma were independently associated with tumor recurrence (Table 3). Moreover, the most important variable related to recurrence of benign meningiomas were co-expression of MIF and MMP9 in tumor with a hazard ratio of $18.383(\mathrm{P}=0.021)$ (Table 4). Other clinical parameters such as age, gender, microvessels density, and tumor size exhibited no association with the tumor recurrence of patients.
Table 2. Kaplan-Meier analysis for recurrence-free time of patients with meningiomas

\begin{tabular}{|c|c|c|}
\hline Characteristics & $\begin{array}{l}\text { Mean recurrence } \\
\text { free time (months) }\end{array}$ & $\begin{array}{l}P \text { val- } \\
\text { ues* }\end{array}$ \\
\hline Age (years) & & 0.238 \\
\hline$<45(\mathrm{n}=33)$ & $71.09 \pm 20.60$ & \\
\hline$\geq 45(n=34)$ & $60.05 \pm 20.66$ & \\
\hline Gender & & 0.334 \\
\hline Male $(\mathrm{n}=17)$ & $65.82 \pm 22.08$ & \\
\hline Female $(\mathrm{n}=50)$ & $65.38 \pm 21.14$ & \\
\hline Histological grade & & 0.001 \\
\hline benign (WHO I) $(\mathrm{n}=57)$ & $69.75 \pm 18.33$ & \\
\hline non-benign (WHO II-III) (n=10) & $41.20 \pm 20.94$ & \\
\hline Tumor size & & 0.138 \\
\hline Small $(n=43)$ & $62.46 \pm 21.37$ & \\
\hline Large $(n=24)$ & $70.91 \pm 20.23$ & \\
\hline Invasion of adjacent tissues & & 0.001 \\
\hline No $(n=47)$ & $70.21 \pm 18.06$ & \\
\hline Yes $(n=20)$ & $54.40 \pm 24.28$ & \\
\hline MIF expression & & 0.052 \\
\hline High expression $(\mathrm{n}=39)$ & $60.21 \pm 20.71$ & \\
\hline Low expression $(n=28)$ & $72.85 \pm 18.45$ & \\
\hline MMP9 expression & & 0.115 \\
\hline High expression $(n=37)$ & $61.48 \pm 23.48$ & \\
\hline Low expression $(n=30)$ & $70.43 \pm 17.16$ & \\
\hline Co-expression of MIF and MMP9 & & 0.005 \\
\hline Dual high-expression $(\mathrm{n}=28)$ & $57.82 \pm 22.39$ & \\
\hline Non-dual high-expression $(\mathrm{n}=39)$ & $71.00 \pm 18.73$ & \\
\hline $\begin{array}{l}\text { MIF and MMP9 in benign menin- } \\
\text { giomas }\end{array}$ & & 0.005 \\
\hline Dual high-expression $(\mathrm{n}=23)$ & $65.13 \pm 16.17$ & \\
\hline Non-dual high-expression $(\mathrm{n}=34)$ & $72.88 \pm 19.26$ & \\
\hline
\end{tabular}

Table 3. Cox regression model for multivariate analyses of recurrence factor in total meningiomas.

\begin{tabular}{llll}
\hline Variable & Hazard ratio & $95 \%$ confidence interval & $P$ value \\
\hline Age $(<45$ vs. $\geq 45)$ & 0.968 & $0.265-3.536$ & 0.961 \\
Gender (male vs. female) & 0.654 & $0.121-3.528$ & 0.621 \\
Histological grade (benign vs. non-benign) & 49.033 & $4.349-552.738$ & 0.002 \\
Tumor size (small vs. large) & 0.327 & $0.076-1.399$ & 0.131 \\
Invasion of adjacent tissues (no vs. yes) & 2.326 & $0.465-11.616$ & 0.303 \\
Angiogenesis (MVD value) & 0.920 & $0.834-1.014$ & 0.095 \\
Dual high MIF and MMP9 expression (no vs. yes) & 37.766 & $3.856-369.816$ & 0.002 \\
\hline
\end{tabular}

Table 4. Cox regression model for multivariate analyses of recurrence factor in benign meningiomas

\begin{tabular}{llll}
\hline Variable & Hazard ratio & $95 \%$ confidence interval & $P$ value \\
\hline Age $(<45$ vs. $\geq 45)$ & 1.201 & $0.211-6.824$ & 0.836 \\
Gender (male vs. female) & 0.265 & $0.017-3.963$ & 0.335 \\
Tumor size (small vs.large) & 0.196 & $0.029-1.324$ & 0.094 \\
Invasion of adjacent tissues (no vs. yes) & 1.749 & $0.268-11.407$ & 0.558 \\
Angiogenesis (MVD value) & 0.882 & $0.754-1.032$ & 0.119 \\
Dual high MIF and MMP9 expression (no vs. yes) & 18.383 & $1.528-221.108$ & 0.021 \\
\hline
\end{tabular}



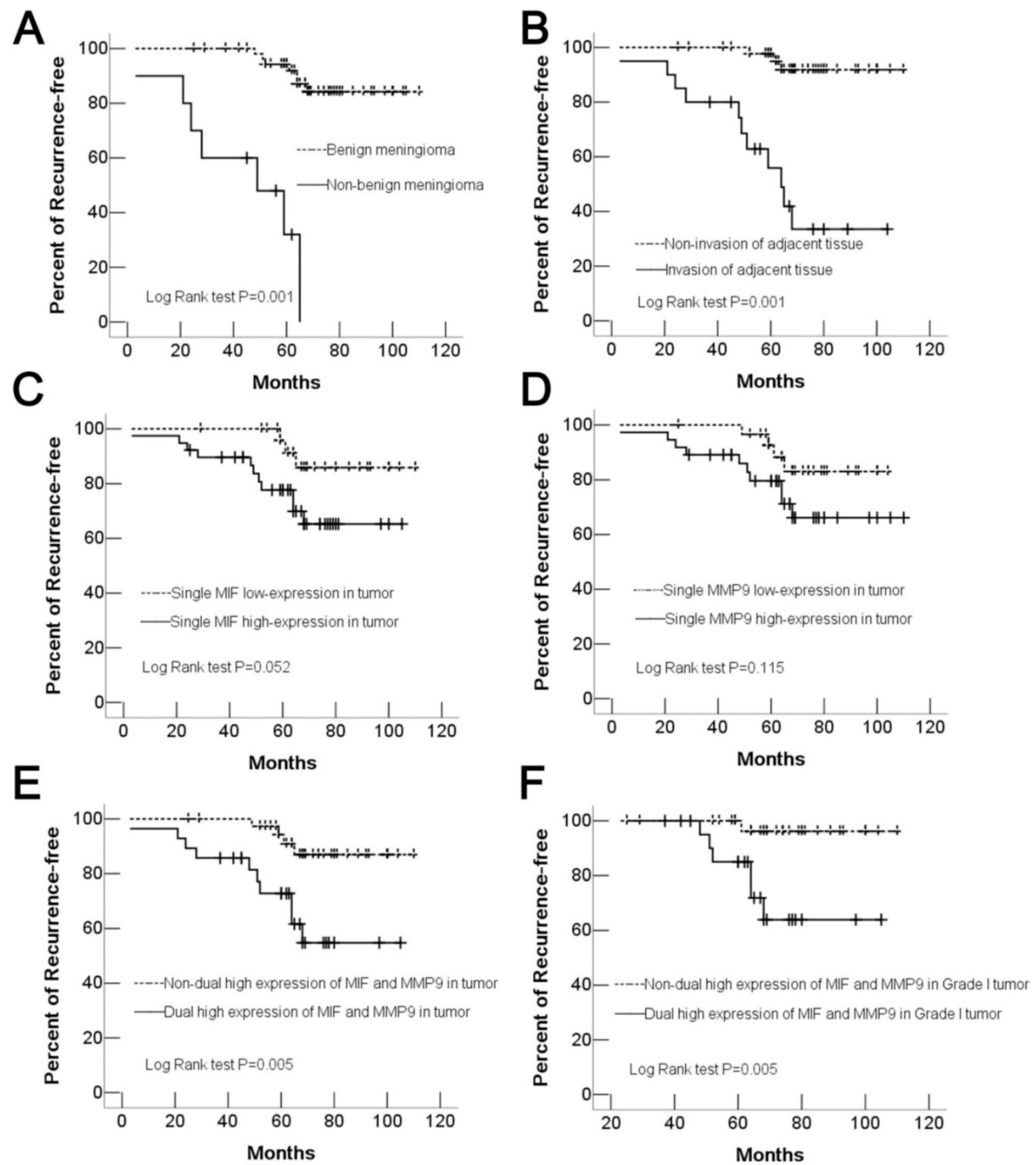

Figure 2. Kaplan-Meier recurrence analyses of meningioma patients. (A) Kaplan-Meier curve showed that patients with non-benign meningioma (grade II and III) have lower recurrence-free rate than those with benign one (grade I). (B) A significant difference in recurrence-free rate was found between tumor with or without invasion of adjacent tissues. There was no significant difference in recurrence-free rate was found between patients with single high and low MIF expression in tumor (C), or single high and low MMP9 expression in tumor (D). (E) Patients with dual high-expression of MIF and MMP9 in tumor had significantly lower recurrence-free rate than those with non-dual high-expression of proteins in tumor. $(\mathrm{F})$ In benign meningiomas, dual high-expression of MIF and MMP9 in tumor was an important factor to influence the recurrence-free rate of patients.

\section{Discussion}

Although certain prognostic factors (Simpson grade, histological grade, and angiogenesis) have been well-established as indicators of meningiomas, the specific factors responsible for tumor recurrence, in particularly, recurrence of benign meningiomas, have not been identified. The present study has shown that MIF and MMP9 may play critical roles in the mechanism of tumor recurrence in meningiomas. MIF is known to promote angiogenesis and modulate cell migration $[13,26]$. In the current study, the result 
of the relationship between MIF high expression and MVD of tumor indicated that MIF might be a possible mediator to contribute to the neovascularization of meningioma and influence the recurrence of meningiomas, because the amount of tumour vascularization has been proposed to affect the degree of cerebral edema and also disease recurrence [24]. However, single MIF high expression in tumors did not affect the time to tumor recurrence of patients independently. A positive correlation with tumor invasion and a significantly shortened recurrence-free time occurred in patients only when the tumor dual high-expressed MIF and MMP9. These results indicate that MIF high expression in tumor is not an independent factor to influence the recurrence of meningioma patients.

MMP9 has been reported to be markedly associated with invasion and metastasis in human cancers [27-28]. The mechanism of meningiomas to invade surrounding structures is poorly understood but the expression of metalloproteinases may be involved in this process, including MMP2 and MMP9 [29-31]. Invasion of surrounding structures by meningiomas also depends on proteases, to degrade the ECM and promote cells migration. MMP9 has been showed a tendency to increase with increasing tumor grade [19, 32], and reported to participate in the angiogenic process in a time-dependent manner [33]. This may be related to the increase in angiogenesis present in higher-grade tumors. In the current study no correlation was found between MMP9 expression and tumor invasion or histological grade although MMP9 showed close correlation to MVD of tumor like similar findings reported in a previous study [19]. These results indicated that increased MMP9 might be one way by which tumor cells achieve greater mobility, but was not an independent factor to influence the prognosis of meningiomas. An interesting tendency towards high MIF expression cases in relation to higher MMP9 expression might be an important reason to elucidate the cooperative effect of MIF and MMP9 on the tumor recurrence of meningioma patients. There was a significantly shorter recurrence-free time only in meningioma patients with MIF and MMP9 dual high expression. In addition, strong co-expression of MIF/MMP9 was closely related to tumor invasion and acted as an independent recurrence factor in multivariate analysis. MIF is a known stimulus for MMP9 expression in inflammatory cells and varied tumor cells [21-22]. Therefore, we postulated that increased MIF and MIF-induced MMP9 production might promote the tumor recurrence through the way of both tumor cell migration and proliferation in meningiomas, increased co-expression of MIF and MMP9 could be a valuable marker for prognostic prediction in meningiomas. Of course, the further study will be necessary to confirm its relationship in a large number of meningiomas and in vitro studies.

There are conflicting results regarding the relationship between tumor recurrence and invasion of surrounding structures by meningiomas. Olmsted et al. considered that meningiomas causing osteolysis and extending into the soft tissue should be characteristics of malignant even if their initial histology seems benign [34]. Ildan et al. supported this point because their result showed the presence of osteolysis increased the risk of recurrence in meningiomas [24]. However, Alvarez et al did not support this result because they did not find this correlation in their series [35]. In our study, $2(22.2 \%)$ of 9 benign cases of meningioma with skull or dura mater invasion recurred, but $100 \%$ recurrence occurred in 2 benign cases with invasion of brain parenchyma. We postulated that this may be related to strong edema induced by the adherence of tumor to the surrounding brain tissue [36], because several studies have found peritumoral edema was to be strongly correlated with tumor recurrence regardless their histological grade $[24,37]$. Although the presence of tumor invasion showed a correlation with recurrence in univariate analysis, it failed to show an independent predictor for time to recurrence in the multivariate analysis. Comparing with dura mater invasion, invasion of brain parenchyma by meningiomas might be more valuable predictive factor for tumor recurrence.

It has been reported that large tumors were associated with higher incidence of tumor infiltration and adherence to arachnoid membrane and the adjacent brain tissue than small tumors [36, 38]. A recent study revealed that increasing tumor size correlated closely with the increasing recurrence rate [24]. However, in our study, there was no statistical correlation between tumor size and other clinicopathological factors, including tumor invasion, recurrence and relevant proteins expression. Further investigations are necessary to reveal this correlation with larger series.

Our results repeatedly confirm that histological grade is so far the most valuable predictive factor of recurrence in meningiomas [39]. The recurrence rate of non-benign meningiomas was significantly higher than that benign meningioma. Regrettably, we have not stratified further the incidence of tumor recurrence in grade II and grade III, and correlate them with proteins expression respectively because of lack of samples. In our study, histological grade failed to predict the recurrence of grade I meningiomas. In fact, 
despite the recent rapid proliferation of literature on the early prediction of meningioma recurrence, a reliable factor on the prognostic value of the recurrence rate in histological benign meningiomas has not yet been identified. In the current study, co-expression of MIF and MMP9 exhibited strongly their correlation with tumor recurrence of benign meningiomas. That indicated increased co-expression of MIF and MMP9 in grade I tumor might be a useful biomarker for higher incidence of tumor recurrence. Together with histological grade, MIF and MMP9 expression status in meningiomas could help clinicians improve the prognostic prediction, and more importantly, adopt corresponding therapeutic approaches to treat theses tumors even if their initial histology seems benign.

In conclusion, to our knowledge, the present data for the first time provide a correlation between co-expression of MIF and MMP9 in tumor and tumor recurrence of meningiomas. Our findings suggest that co-expression of MIF and MMP9 in tumor might be a valuable predictor for recurrence of benign meningiomas.

\section{Competing Interests}

The authors have declared that no competing interest exists.

\section{References}

1. Perry A, Louis DN, Scheithauer BW. Meningiomas. In: Loui DN, Ohgaki $\mathrm{H}$, Wiestler OD, eds. WHO classification of tumors of the central nervous system. Lyon: IARC Press. 2007:164-172.

2. Perry A, Stafford SL, Scheithauer BW, et al. Meningioma grading: an analysis of histologic parameters. Am J Surg Pathol 1997; 21: 1455-65.

3. Whittle IR, Smith C, Navoo P, Collie D. Meningiomas. Lancet 2004; 363:1535-43.

4. Bach JP, Rinn B, Meyer B, et al.: Role of MIF in inflammation and tumorigenesis. Oncology 2008; 75:127-33.

5. Feng $\mathrm{XP}, \mathrm{Yi} \mathrm{H}, \mathrm{Li} \mathrm{MY}$, et al. Identification of biomarkers for predicting nasopharyngeal carcinoma response to radiotherapy by proteomics. Cancer Res 2010; 70:3450-62.

6. Krockenberger M, Engel JB, Kolb J, et al. Macrophage migration inhibitory factor expression in cervical cancer. J Cancer Res Clin Oncol 2010; 136:651-7.

7. Rendon BE, Roger T, Teneng I, et al. Regulation of human lung adenocarcinoma cell migration and invasion by macrophage migration inhibitory factor. J Biol Chem 2007; 282:29910-8.

8. $\mathrm{Xu} \mathrm{X}$, Wang B, Ye C, et al. Overexpression of macrophage migration inhibitory factor induces angiogenesis in human breast cancer. Cancer Lett 2008; 261:147-57.

9. Bach JP, MA OD, Balzer-Geldsetzer M, et al. The role of macrophage inhibitory factor in tumorigenesis and central nervous system tumors. Cancer 2009; 115:2031-40.

10. Li GQ, Xie J, Lei XY, Zhang L. Macrophage migration inhibitory factor regulates proliferation of gastric cancer cells via the PI3K/Akt pathway. World J Gastroenterol 2009; 15:5541-8.

11. Lue H, Thiele M, Franz J, et al. Macrophage migration inhibitory factor (MIF) promotes cell survival by activation of the Akt pathway and role for CSN5/JAB1 in the control of autocrine MIF activity. Oncogene 2007; 26:5046-59.

12. Markert JM, Fuller CM, Gillespie GY, et al. Differential gene expression profiling in human brain tumors. Physiol Genomics 2001; 5:21-33.

13. Munaut C, Boniver J, Foidart JM, Deprez M. Macrophage migration inhibitory factor (MIF) expression in human glioblastomas correlates with vascular endothelial growth factor (VEGF) expression. Neuropathol Appl Neurobiol 2002; 28:452-60.
14. Meyer-Siegler KL, Iczkowski KA, Leng L, Bucala R, et al. Inhibition of macrophage migration inhibitory factor or its receptor (CD74) attenuates growth and invasion of DU-145 prostate cancer cells. J Immunol 2006; 177:8730-9.

15. Ren $\mathrm{Y}, \mathrm{Chan} \mathrm{HM}$, Fan J, et al. Inhibition of tumor growth and metastasis in vitro and in vivo by targeting macrophage migration inhibitory factor in human neuroblastoma. Oncogene 2006; 25:3501-8.

16. Curran S, Murray GI. Matrix metalloproteinases: molecular aspects of their roles in tumour invasion and metastasis. Eur J Cancer 2000; 36:1621-30.

17. Kim TD, Song KS, Li G, et al. Activity and expression of urokinase-type plasminogen activator and matrix metalloproteinases in human colorectal cancer. BMC Cancer 2006; 6:211.

18. Mendes O, Kim HT, Stoica G. Expression of MMP2, MMP9 and MMP3 in breast cancer brain metastasis in a rat model. Clin Exp Metastasis 2005; 22:237-46.

19. Panagopoulos AT, Lancellotti CL, Veiga JC, de Aguiar PH, et al. Expression of cell adhesion proteins and proteins related to angiogenesis and fatty acid metabolism in benign, atypical, and anaplastic meningiomas. J Neurooncol 2008; 89:73-87.

20. Yu X, Lin SG, Huang XR, et al. Macrophage migration inhibitory factor induces MMP-9 expression in macrophages via the MEK-ERK MAP kinase pathway. J Interferon Cytokine Res 2007; 27:103-9.

21. Dumitru CA, Gholaman H, Trellakis S, et al. Tumor-derived macrophage migration inhibitory factor modulates the biology of head and neck cancer cells via neutrophil activation. Int J Cancer 2011; 129:859-69.

22. Li Z, Ren $Y, W u Q C$, et al. Macrophage migration inhibitory factor enhances neoplastic cell invasion by inducing the expression of matrix metalloproteinase 9 and interleukin- 8 in nasopharyngeal carcinoma cell lines. Chin Med J (Engl) 2004; 117:107-14.

23. Simpson D. The recurrence of intracranial meningiomas after surgical treatment. J Neurol Neurosurg Psychiatry 1957; 20:22-39.

24. Ildan F, Erman T, Göçer AI, et al. Predicting the probability of meningioma recurrence in the preoperative and early postoperative period: a multivariate analysis in the midterm follow-up. Skull Base 2007; 17:157-71.

25. Pistolesi S, Boldrini L, Gisfredi S, et al. Angiogenesis in intracranial meningiomas: immunohistochemical and molecular study. Neuropathol Appl Neurobiol 2004; 30:118-25.

26. Bacher M, Schrader J, Thompson N, et al. Up-regulation of macrophage migration inhibitory factor gene and protein expression in glial tumor cells during hypoxic and hypoglycemic stress indicates a critical role for angiogenesis in glioblastoma multiforme. Am J Pathol 2003; 162:11-7.

27. Deryugina EI, Quigley JP. Pleiotropic roles of matrix metalloproteinases in tumor angiogenesis: contrasting, overlapping and compensatory functions. Biochim Biophys Acta 2010; 1803:103-20.

28. Zheng H, Takahashi H, Murai $Y$, et al. Expressions of MMP-2, MMP-9 and VEGF are closely linked to growth, invasion, metastasis and angiogenesis of gastric carcinoma. Anticancer Res 2006; 26:3579-83.

29. Okuducu AF, Zils U, Michaelis SAM, et al. Ets-1 is up-regulated together with its target gene products matrix metalloproteinase- 2 and matrix metalloproteinase- 9 in atypical and anaplastic meningiomas. Histopathology 2006; 48:836-45.

30. Okada M, Miyake K, Matsumoto Y, et al. Matrix metalloproteinase-2 and matrix metalloproteinase- 9 expressions correlate with the recurrence of intracranial meningiomas. J Neurooncol 2004; 66:29-37.

31. Von Randow AJU, Schindler S, Tews DS. Expression of extracellular matrix degrading proteins in classic, atypical and anaplastic meningiomas. Pathol Res Pract 2006; 202:365-72.

32. Paek SH, Kim CY, Kim YY, et al. Correlation of clinical and biological parameters with peritumoral edema in meningioma. J Neurooncol 2002; 60:235-45.

33. Kiran MS, Kumar VBS, Viji RI, Sudhakaran PR. Temporal relationship between MMP production and angiogenic process in HUVECS. Cell Biol Int 2006; 30:704-13.

34. Olmsted WW, McGee TP. Prognosis in meningioma through evaluation of skull bone patterns. Radiology 1977; 123:375-7.

35. Alvarez F, Roda JM, Perez Romero M, et al. Malignant and atypical meningiomas: a reappraisal of clinical, histological, and computed tomographic features. Neurosurgery 1987; 20:688-94.

36. Ide $\mathrm{M}$, Jimbo $\mathrm{M}, \mathrm{Kubo} \mathrm{O}$, et al.: Peritumoral brain edema and cortical damage by meningioma. Acta Neurochir Suppl (Wien) 1994; 60:369-72.

37. Christensen D, Laursen H, Klinken L. Prediction of recurrence in meningiomas after surgical treatment: a quantitative approach. Acta Neuropathol (Berl) 1983; 61:130-4. 
38. Bitzer M, Wöckel L, Morgalla M, et al. Peritumoural brain oedema in intracranial meningiomas: influence of tumour size, location and histology. Acta Neurochir (Wien) 1997; 139:1136-42.

39. Adegbite AB, Khan MI, Paine KWE, Tan LK. The recurrence of intracranial meningiomas after surgical treatment. J Neurosurg 1983; 58:51-6. 